\title{
Modulation of Corticospinal Motor Tract Excitability during a Fine Finger Movement under Psychological Pressure: A TMS Study
}

\author{
Yoshifumi Tanaka1, Kozo Funase ${ }^{2}$, Hiroshi Sekiya² and Takayuki Murayama ${ }^{3}$ \\ ${ }^{1}$ Faculty of Education and Regional Studies, University of Fukui \\ 3-9-1 Bunkyo, Fukui, 910-8507 Japan \\ tnk@u-fukui.ac.jp \\ ${ }^{2}$ Graduate School of Integrated Arts and Sciences, Hiroshima University \\ 1-7-1 Kagamiyama, Higashihiroshima, Hiroshima, 739-8521 Japan \\ ${ }^{3}$ Health Service Center, Kanazawa University \\ Kakumamachi, Kanazawa, Ishikawa, 920-1192 Japan \\ [Received October 31, 2011; Accepted March 1, 2012; Published online April 19, 2012]
}

\begin{abstract}
In the present study, the influence of psychological pressure on corticospinal motor tract (CST) excitability was investigated using monophasic transcranial magnetic stimulation (TMS) during a fine finger movement. Nine participants performed a spatiotemporal tracing task using a small laser pointer attached to the right index finger. After seven acquisition trials, they performed two non-pressure trials followed by two pressure trials. A stress was successfully induced; state anxiety, heart rates, and galvanic skin response all increased under pressure. In terms of motor evoked potential (MEP) in the first dorsal interosseous muscle, increases in amplitude and decreases in latency were shown under pressure. Moreover, whereas background electromyography (bEMG) before TMS failed to increase significantly under pressure, the ratio of MEP amplitude to bEMG did increase under pressure. In addition, there was a significant positive correlation between increased CST excitability and decreased performance accuracy from non-pressure to pressure trials. These results suggest that psychological pressure can facilitate the excitatory level of CST during performance of a fine motor skill and that CST excitation under pressure can relate to decreased performance.
\end{abstract}

Keywords: stress, arousal, transcranial magnetic stimulation, motor evoked potential, electromyography

\section{Introduction}

When participating in activities such as important sports competitions and performances witnessed by large audiences, people are expected to do their best under psychological pressure. Overcoming these circumstances is an important concern that many athletes face. Psychological pressure is defined as "any factor, or combination of factors that increases the importance of performing well on a particular occasion"' (Baumeister, 1984). Previous studies have examined three aspects related to the effects of psychological pressure on motor skills, namely, psychological (e.g., Liao and Masters, 2002; Martens and Landers, 1970), physiological (e.g., Mullen et al., 2005; Salvador et al., 2003), and behavioral properties (e.g., Court et al., 2005; Higuchi et al., 2002).
A model has been proposed that accounts for these changes from the perspective of neurophysiology (Hatfield, 2007). According to this model, psychological pressure causes changes in excitatory levels of the prefrontal and temporal cortex, involved in higher-level cognition, as well as changes in the amygdala and anterior cingulate cortex, involved in emotional processing. In turn, these changes affect areas in the higher central nervous system, such as the basal ganglia, thalamus, supplementary motor area, pre-motor cortex and primary motor area. In addition, changes occur in the excitatory level of the spinal tract, which acts as the main descending pathway for voluntary movements. All such changes lead to alterations in the kinematic domain and motor unit activities. Although a number of studies have examined motor control in kinematic (e.g., Hasegawa et al., 2011; Tanaka and Sekiya, 2010) and elec- 
tromyography (EMG) (e.g., Yoshie et al., 2008, 2009) functions in subjects under psychological pressure, few studies have investigated these functions from a neurophysiological perspective.

Recently, this model was corroborated in a study in which electroencephalogram (EEG) activity was recorded while participants performed simulated driving (Murray and Janelle, 2007). Additionally, one pilot study used monophasic transcranial magnetic stimulation (TMS) to examine the influence of psychological pressure on the excitatory level of the corticospinal motor tract (CST), including primary motor areas of the brain (Rollnik et al., 2000). TMS was used to investigate the degree of motor output. The amplitude and latency of motor evoked EMG potentials (MEP) for the target muscle were recorded while examining the excitatory level of the CST by stimulating the primary motor area. Rollnik et al. reported increases in MEP amplitude and decreases in MEP latency for most participants after the participants played a video game under pressure induced by a competitive situation. However, in their study, a homogenous pattern of CST excitability under pressure did not (significantly) occur. In addition, while MEP variables were measured after task performance by Rollnik et al., measuring CST excitability when an individual is performing motor skills may be more reflective of realistic sport contexts. To date, there have been no published reports of CST excitability during on-line motor skill performance under pressure.

The current study used monophasic TMS to investigate the excitatory level of the CST when executing a fine motor skill under psychological pressure. We hypothesized that increased CST excitability (i.e., an increase in MEP amplitude and a decrease in MEP latency) would be observed when participants performed a fine motor task under pressure. It was predicted that emotional changes and the effects of physiological arousal under pressure would facilitate the excitatory level of the CST. Some studies have reported that emotional changes, along with increased arousal levels, relate to the excitatory level of the CST. For example, positive and/or negative emotional states that are induced by pleasant or unpleasant stimuli elevate excitatory levels of the primary motor (e.g., Coombes et al., 2009; van Loon et al., 2010) and supplementary motor areas (Oliveri et al., 2003). Similar to these previous studies using emotional stimuli, psychological pressure would be expected to facilitate CST excitability.

Additionally, in many previous studies examining motor control and behavior under psychological pressure, performance outcomes of motor skills have been investigated. These studies have reported varied results, ranging from performance decrements (e.g., Williams et al., 2002; Yoshie et al., 2009) and maintenance (e.g., Higuchi et al., 2002; Tanaka and Sekiya, 2010) to improvements (e.g., Ehrlenspiel et al., 2010; Tanaka et al., 2011). In line with these various findings, prior research shows that performance changes under pressure relate to changes in psychological, physiological, and kinematic factors. For example, Martens and Landers (1970) showed an inverted-U pattern between physiological arousal and performance accuracy on a tracking task. Moreover, other studies indicate a negative linear correlation between increased internal focus of attention and performance accuracy of a golf-putting task (Tanaka and Sekiya, 2011), a positive linear correlation between increased quieteye period and shooting accuracy in a biathlon (Vickers and Williams, 2007), and a positive linear correlation between increased compensatory control among kinematic variables and performance accuracy in a ball bouncing task (Ehrlenspiel et al., 2010). However, the relationship between motor performance and CST excitability under pressure has never been examined.

Given the aforementioned background, in the present study, the linear correlations among changes in all dependent variables from non-pressure to pressure circumstances were calculated in order to examine the relationship between psycho-physiological aspects, including state anxiety, physiological arousal, CST excitability, and EMG activity on the one hand, and performance accuracy of a fine motor task under pressure on the other hand. For consistency and ease of data recording, the MEPs from hand muscles have been recorded in previous studies using the monophasic TMS technique (e.g., Coombes et al., 2009; van Loon et al., 2010). Similarly, in the present study, a spatiotemporal tracing task was chosen as an experimental movement task. Specifically, a small laser pointer was attached to participants' right index finger while tracing a target circle. The MEP from the first dorsal interosseous (FDI) muscle was recorded during the movement task. 


\section{Method}

\subsection{Participants}

Nine right-handed university students (3 males and 6 females) aged 18-22 (mean $\pm \mathrm{SD}=19.44 \pm$ 1.42) participated in the study. All participants denied the presence of epilepsy or other neurological disorder. Informed consent was obtained from all participants prior to study participation. The experiment was approved by the ethics committee of Hiroshima University.

\subsection{Tasks}

The experimental setting for the movement task is shown in Figure 1. While participants sat in a chair, a small laser pointer $(40 \mathrm{~g})$ was taped to their right index finger. Each participant's right elbow and right wrist were stabilized to the table. The target had three concentric circles with different diameters of 13,15 , and $17 \mathrm{~cm}$, respectively.

The task required participants to smoothly move the laser light point projected by the laser pointer in a clock-wise motion along a circle with a $15 \mathrm{~cm}$ diameter using their right index finger. They were instructed to keep the laser light point as close to the outline of the circle as possible by maintaining the point within a $2 \mathrm{~cm}$ band that was outside the $13 \mathrm{~cm}$ diameter circle and inside the $17 \mathrm{~cm}$ diameter circle. Participants heard a beep which was generated every second. They were instructed to move the light point by $1 / 8$ of the circle every second by moving the pointer in time with the beeps. The circular movement

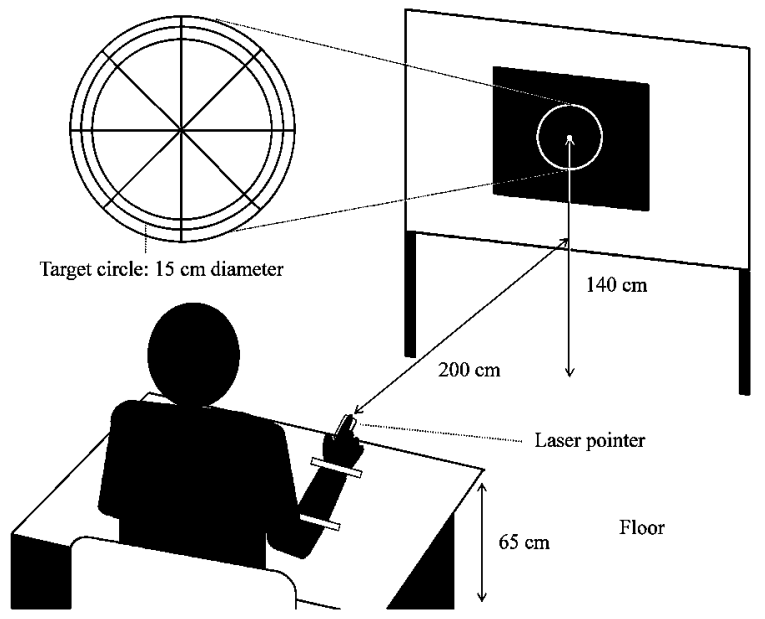

Figure 1 Apparatus used for the spatiotemporal tracing task. started at the highest (vertical) point of the circle $\left(0^{\circ}\right)$. The task was complete when the laser light point completed the circle 15 times. Each trial was 2 minutes in length (i.e., 15 times around the circle, with each cycle taking $8 \mathrm{~s}$ ). Participants performed one practice trial prior to beginning the experimental trial. During the practice trial, an instruction to move the laser light point smoothly was added if they performed a saccadic movement with the beeps. The task was recorded by a digital video camera (DCR-TRV70K, Sony Corporation, Japan) located behind the participants. Video recordings were at 30 $\mathrm{Hz}$ sampling frequency.

\subsection{Induction and recording of physiological in- dices}

A figure-of-eight coil with a $70 \mathrm{~mm}$ diameter was connected to a magnetic stimulator (model 200, Magstim Company, UK) to induce MEP. The coil was attached to the optimal control region of the FDI muscle in the motor area of the left hemisphere and monophasic TMS was administered during the tasks to induce MEP from the right hand's FDI muscle. The coil was placed tangentially to the scalp, with the handle pointing backward and rotated approximately 30 degrees away from the mid-sagittal line. Each participant wore a swimming cap during the experiment. The position of the coil was marked on the swimming cap so that the coil's position could be monitored and maintained during the experiment.

Active motor threshold of MEP was defined as the lowest stimulus intensity that consistently evoked MEP in the FDI muscle of the right hand, with an amplitude of at least $50 \mu \mathrm{V}$ and probability of at least $50 \%$. The mean active motor thresholds for MEP of participants were $39.78 \pm 6.61 \%$ of the maximum output of TMS (2.0 tesla). In the experiment, the TMS administered to a given participant was 1.2 to 1.3 times higher $(48.22 \pm 8.14 \%)$ than the participant's MEP active motor threshold. This TMS intensity was determined such that MEP amplitude reached approximately $1 \mathrm{mV}$. EMG was amplified and extracted by $\mathrm{Ag}-\mathrm{AgCl}$ bipolar surface electrodes $(1.0 \mathrm{~cm}$ diameters) recording with a bandwidth of $5 \mathrm{~Hz}$ to $3 \mathrm{kHz}$ (model 7S12, NEC San-ei Co. Ltd., Japan). Heart rate (HR) and galvanic skin response (GSR) were measured using a HR monitor (POLAR BANTAGE XL, Canon Inc., Japan) and a 
GSR amplifier (ML 116, AD Instruments Pty. Ltd., Australia), respectively. The HR monitor transmitter was attached to the participant's chest and a receiver was attached to the left wrist. The GSR sensor was attached to the left middle finger phalanx distalis on the palm side. A computer recorded the MEP, EMG, and GSR obtained with a sampling frequency of $10 \mathrm{kHz}$ (Power Lab 4/30, Chart 5 for Windows, AD Instruments Pty. Ltd., Australia).

\subsection{Procedure}

The HR monitor was attached and a participant was seated in the chair. The equipment for measuring MEP, EMG, and GSR was then attached. In order for participants to be familiarized with TMS administrations when performing the movement task, the task was repeated twice with TMS administrations as a pre-test. In one of the pre-test trials, TMS was administered when the laser light point was at 270 degrees in the first round through the circle. Eleven additional administrations of TMS followed at every 450 degrees. Three administrations of TMS were presented at $0,90,180,270$ degrees for a total of 12 TMS administrations. In another trial of the pre-test, the first administration of TMS occurred at 225 degrees in the first round, followed by 11 more administrations of TMS every 450 degrees. This consisted of three administrations of TMS at $45,135,225$, and 315 degrees for a total of 12 TMS administrations. When involuntary movements occurred in the FDI muscle because of TMS administrations and the laser light point moved outside the target, participants were instructed to move the laser light point back to where it was before the involuntary movements occurred and restart the task after the beeps. After these pre-test trials, participants repeated the task in five more acquisition trials without TMS administrations in order to become familiar with the task.

Next, participants received four test trials. They repeated the task two times without pressure (nonpressure test). In these two trials, TMS was administered using the same method as the pre-test. Both HR and GSR were recorded during these trials and state anxiety was measured between the two trials using the revised State-Trait Anxiety InventoryForm-JYZ Y1 (STAI; Hidano et al., 2000). The last two test trials (pressure test) involved recording the participant's performance under psychological pres- sure.

The following instruction was given to the participants prior to final two test trials: "In the following two trials, I will praise you if the laser light point moves out of the outer and inner boundaries fewer than seven times in both trials. However, if the laser light point moves out of the boundaries more than nine times, you will have to start the experiment over." In these trials, TMS was administered in a manner similar to that of the pre-test and non-pressure test and HR, GSR, and state anxiety were measured. After participants completed the pressure-test trials, they were debriefed about the experiment and informed that the instruction about starting over if the laser point moved out of the boundaries was a false instruction.

In each of these tests, the time of administering TMS differed between the two trials. The order of the two trials was counterbalanced among participants. The order of the non-pressure and pressure tests could not be counterbalanced in the present study since it was necessary to tell the participants that they had to start the experiment over after the non-pressure test. Previous studies (e.g., Beilock and Carr, 2001; Cooke et al., 2010; Tanaka and Sekiya, 2010) have adopted this experimental procedure in order to prevent reduced motivation during the non-pressure condition after participants had already experienced the pressure condition.

\subsection{Dependent variables}

\subsubsection{Manipulation check of pressure}

To determine the psychological effects of pressure, state anxiety was measured using the STAI. HR was recorded every 5 seconds and GSR was measured to assess physiological changes. The mean HR and GSR during each trial were calculated.

\subsubsection{MEP and EMG}

Amplitude (peak-to-peak) and latency of MEP, induced by TMS, was calculated for each stimulus. Latency was based on the time that the MEP waveform reached $0.1 \mathrm{mV}$. Integrated background EMG (bEMG) was calculated for the $100 \mathrm{~ms}$ duration before TMS was administered. Because MEP amplitude becomes larger linearly if muscle tension increases gradually during voluntary movement (Capaday, 1997; Devanne et al., 1997; Hasegawa et al., 2001; Kasai, 1993), it is necessary to ensure that 
any changes in MEP amplitude are not simply due to EMG activity under pressure. To adjust for this possibility, the ratio of MEP amplitude to bEMG (MEP amplitude/bEMG) was also calculated for each stimulus. This ratio has been used to measure CST excitability accompanied with muscle contractions in prior research (e.g., Kazennikov et al., 2008; Yahagi et al., 2005).

For MEP and EMG variables, standardized percentages were calculated by dividing the values obtained from the non-pressure and pressure tests by values obtained from the pre-test with identical timing due to considerations of inter-personal large variability. Such standardized scores were used in previous studies to measure MEP and EMG (e.g., Kazennikov et al., 2008; Hayashi et al., 2003; van Loon et al., 2010).

\subsubsection{Performance}

Using motion analysis software (Frame-DIAS for Windows, DKH, Japan), the laser light point and the circle with a $15 \mathrm{~cm}$ diameter in the target of the video recordings were converted to coordinates in a two-dimensional representation and the distance between them was calculated. Based on this distance, absolute errors (AE) and absolute constant errors (ACE) were calculated. Variable errors (VE) were calculated as indices of intra-trial variability in the positions of the laser light point using the following formula:

$$
\begin{aligned}
& \operatorname{AE}(m m)=(1 / n) \sum_{i=1}^{n}\left|X_{i}-75 m m\right|, \\
& \mathrm{CE}(m m)=(1 / n) \sum_{i=1}^{n}\left(X_{i}-75 m m\right), \\
& A C E(m m)=|C E|, \\
& V E(m m)=\sqrt{(1 / n) \sum_{i=1}^{n}\left[X_{i}-\bar{X}\right]^{2}}
\end{aligned}
$$

in which $n$ equals number of frames (i.e., the number of data points collected within a single trial), $X_{i}$ is the distance from the center of target, and $X$ is the participant's mean error distance from the target circle in each trial. Two seconds (60 frames) were excluded from the analysis due to involuntary movements in FDI muscle caused by TMS.

\subsection{Data analysis}

Due to established gender differences in state anxi- ety, state anxiety scores from the non-pressure and pressure tests were converted to t-scores based on mean scores and standard deviations for male $(\mathrm{n}=$ $1088)$ and female $(n=1165)$ university students published in the STAI manual (Hidano et al., 2000). A paired t-test was conducted on the standardized state anxiety scores for the non-pressure and pressure tests. The other dependent variables were analyzed using two-factor (two test blocks $\times$ two trials) repeated measures analyses of variance (ANOVAs). Because the order of the non-pressure and pressure tests could not be counterbalanced in the present study, the possibility of an order effect in the significant main effect for trial and the interaction between test and trial cannot be discounted. In order to test the phase difference of TMS administrations, one-factor (eight difference phases) repeated measure ANOVAs were conducted for all MEP and EMG variables. A Bonferroni correction was used for planned pair-wise comparisons. In cases when equal variance could not be postulated for Mauchly sphericity tests, we used a Greenhouse-Geisser correction for degrees of freedom and margin of error.

Percent changes between the non-pressure and pressure tests were calculated for all dependent variables. In order to test the relationships among these variables, Spearman rank-order correlation coefficients $(n=9)$ were calculated for all relationships between the dependent variables. Due to the small sample size, rank-order correlation analysis was used. The significance level for all the analyses was set to less than $5 \%$.

\section{Results}

\subsection{Manipulation check of pressure}

The means and standard deviations of the state anxiety scores, HR, and GSR for both non-pressure and pressure tests are shown in Table 1. A t-test revealed a significant increase in state anxiety, $t(8)=$

Table 1 Means and standard deviations of state anxiety, HR, and GSR during the non-pressure test and the pressure test

\begin{tabular}{lcc}
\hline & Non-pressure test & Pressure test \\
\hline State anxiety & $42.67 \pm 6.71$ & $50.89 \pm 12.86$ \\
State anxiety (t-score) & $46.40 \pm 6.48$ & $54.40 \pm 12.67$ \\
HR (bpm) & $70.11 \pm 7.27$ & $75.11 \pm 8.65$ \\
GSR $(\mu \mathrm{S})$ & $25.21 \pm 9.36$ & $29.59 \pm 8.75$ \\
\hline
\end{tabular}


2.77, $p=.024$. The ANOVA for HR showed a significant main effect of test, $F(1,8)=7.44, \eta^{2}=.48, p$ $=.026$, indicating that HR increased from the nonpressure test to the pressure test. GSR failed to reach significant increase between the non-pressure and the pressure test, $F(1,8)=4.57, \eta^{2}=.36, p=.065$. No other main effects or interactions were significant.

\subsection{MEP and EMG}

Figure 2 illustrates an averaged MEP waveform for each phase of the TMS administration recorded from one participant. As shown in Figure 2, there was increased MEP amplitude and no difference of the bEMG amplitude from the non-pressure (upper waveform) to the pressure test (lower waveform). The means and standard deviations of the MEP and EMG variables for all participants in the non-pressure and pressure tests are shown in Figure 3. The results of an ANOVA for standardized MEP amplitude indicated a main effect of test, $F(1,8)=12.83$, $\eta^{2}=.62, p=.007$, with MEP amplitude greater for the pressure than for the non-pressure test. The ANOVA for standardized MEP latency also showed a significant main effect of test, $F(1,8)=12.89, \eta^{2}$ $=.62, p=.007$, indicating that MEP latency

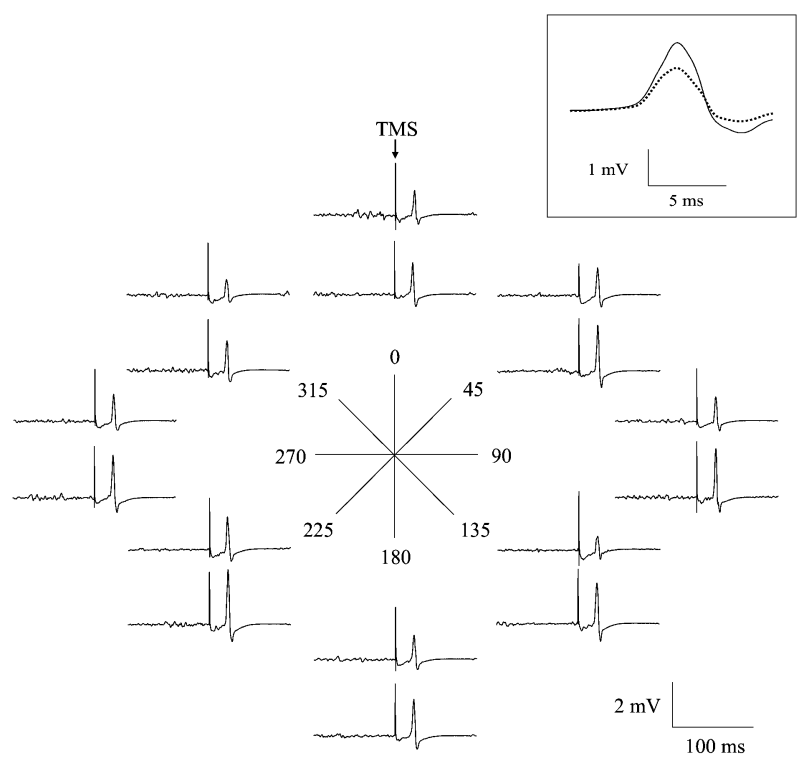

Figure 2 Typical averaged MEP waveforms in each phase, recorded from one participant during the non-pressure test (upper waveform) and pressure test (lower waveform). Data are averaged over three trials. The highlighted panel shows the averaged MEPs of eight different phases during the non-pressure test (dashed line) and the pressure test (solid line). decreased from the non-pressure to the pressure test. Differences in standardized bEMG between the nonpressure and the pressure test failed to reach significance, $F(1,8)=4.15, \eta^{2}=.34, p=.076$. The ANOVA for standardized MEP amplitude/bEMG showed a significant main effect of test, $F(1,8)=$ $5.65, \eta^{2}=.41, p=.045$, indicating that increased MEP amplitude during the pressure test was not due to increased EMG activity. No other main effects or interactions were significant. ANOVAs testing the phase differences of TMS administrations indicated no significant main effects of phase for all MEP and EMG variables.
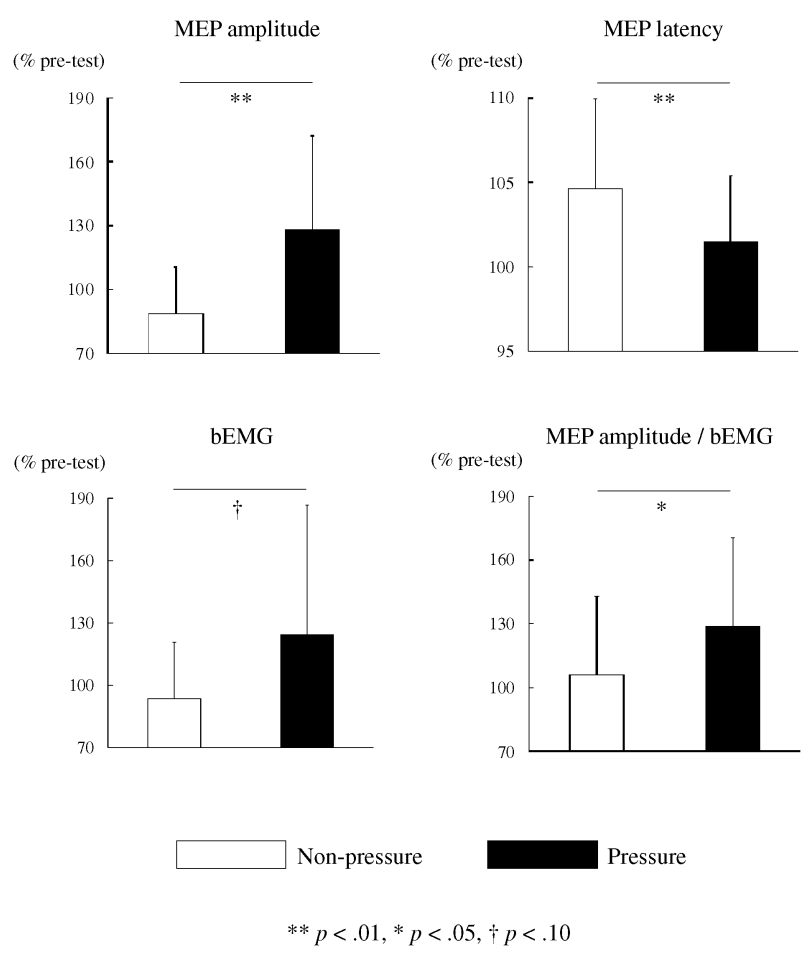

Figure 3 Means and standard deviations of standardized MEP and EMG variables for all participants during the non-pressure and pressure tests.

\subsection{Performance}

Table 2 shows the means and standard deviations of the three types of error scores measured. The ANOVA for AE revealed a significant main effect of test, $F(1,8)=5.63, \eta^{2}=.41, p=.045$, indicating that $\mathrm{AE}$ decreased from the non-pressure test to the pressure test. The difference in VE for the non-pressure and pressure tests failed to reach significance, $F(1,8)=4.04, \eta^{2}=.34, p=.079$. No other main 
Table 3 Matrix of the results of Spearman rank-order correlation coefficients between all dependent variables

\begin{tabular}{|c|c|c|c|c|c|c|c|c|c|c|}
\hline & 1 & 2 & 3 & 4 & 5 & 6 & 7 & 8 & 9 & 10 \\
\hline 1. State anxiety & & $.717^{*}$ & .517 & .283 & -.183 & .300 & -.150 & .083 & .167 & .000 \\
\hline 2. $\mathrm{HR}$ & & & .350 & .433 & -.383 & .433 & .133 & .333 & .500 & -.300 \\
\hline 3. GSR & & & & -.177 & -.017 & .583 & -.617 & -.467 & -.500 & -.083 \\
\hline 4. MEP amplitude & & & & & -.400 & .367 & .433 & .450 & .633 & -.100 \\
\hline 5. MEP latency & & & & & & -.500 & -.067 & -.183 & -.350 & .000 \\
\hline 6. bEMG & & & & & & & -.567 & -.350 & .067 & -.583 \\
\hline 7. MEP amplitude/bEMG & & & & & & & & $.800^{*}$ & .500 & .433 \\
\hline 8. $\mathrm{AE}$ & & & & & & & & & $.683^{*}$ & .383 \\
\hline 9. $\mathrm{ACE}$ & & & & & & & & & & -.300 \\
\hline 10. VE & & & & & & & & & & \\
\hline
\end{tabular}

Note: ${ }^{*} p<.05$.

effects or interactions were significant. The results of an ANOVA for ACE showed no main effects or interaction.

Table 2 Means and standard deviations of indices of performance during the non-pressure test and the pressure test

\begin{tabular}{lcc}
\hline & Non-pressure test & Pressure test \\
\hline AE $(\mathrm{mm})$ & $6.66 \pm 1.14$ & $6.14 \pm 1.00$ \\
ACE $(\mathrm{mm})$ & $5.44 \pm 1.09$ & $5.29 \pm .69$ \\
VE $(\mathrm{mm})$ & $6.16 \pm 1.31$ & $5.47 \pm 1.23$ \\
\hline
\end{tabular}

\subsection{Relationships between dependent variables}

Spearman rank-order correlation coefficients for all dependent variables are shown in Table 3. The coefficient of MEP amplitude/bEMG was significant for $\operatorname{AE}(r=.800, p<.05)$, indicating that less change in CST excitability from the non-pressure to the pressure tests was associated with decreased AE and that increased CST excitability during the pressure test led to increased AE. A significant positive correlation between STAI and HR was found $(r=.717$, $p<.05)$ and the coefficient of ACE was significant for $\operatorname{AE}(r=.683, p<.05)$. No other significant coefficients were found.

\section{Discussion}

In the present study, state anxiety, HR, and GSR were examined in order to investigate the effects of added pressure. These variables all increased during the pressure test, although there was only a trend toward an increase in GSR. These results indicate that the punishment and rewards used in this study induced both psychological and physiological stress in participants. Nonetheless, the stress induced in the study was at a low level, as indicated by a mean standardized state anxiety score of 54 and the mean increase in HR of $5 \mathrm{bpm}$.

Both MEP and EMG amplitudes in the non-pressure test were $89 \%$ and $94 \%$, respectively, compared to variables recorded in the pre-test. It is reasonable to assume that the higher MEP and EMG found in the pre-test was caused by psychological stress in participants who encountered unfamiliar experimental settings at the beginning of the experiment. Furthermore, during the pressure test, the results of MEP amplitude and latency indicated that the level of excitability in the CST increased during the fine motor task, even under a low level of stress. However, it might be argued that changes found could be attributed to an order effect, given that the order of the non-pressure and pressure tests could not be counterbalanced. In the present study, test order was fixed to prevent participants from experiencing reduced motivation. Statistical analyses testing the order effect showed that there were no significant interactions or main effects of trial in the two-way ANOVA, suggesting that changes in the MEP and EMG variables during the pressure test were not due to the order effect.

There was a tendency for an increase in EMG activity under pressure in this study. In prior studies, increased EMG under psychological stress has been found when participants had to perform mental calculations while engaging in a motor task (van Galen et al., 2002; van Loon et al., 2001). Thus, the effects of stress may have been responsible for the tendency of EMG to increase under pressure in this study. Be- 
cause many studies have shown that increases in MEP amplitude are associated with increases in EMG (e.g., Devanne et al., 1997), the increased MEP found in the present study could be attributed to increased muscular activities. However, MEP amplitude/bEMG also increased significantly from the non-pressure to pressure test. On the basis of these results, we conclude that it is likely that changes in the excitatory level of CST observed in this study were not due to motor changes, such as changes in EMG amplitude.

Emotional changes and the effects of physiological arousal could be factors contributing to the increased excitatory level of CST under psychological pressure. Previous studies showed that the excitatory level of CST was greater when pictures and music were presented as opposed to neutral stimuli (e.g., Baumgartner et al., 2007; Hajcak et al., 2007), likely due to the stimulation of positive and/or negative emotions along with increases in arousal level. In addition, it is known that anticipatory anxiety facilitates CST excitation (Oathes et al., 2008). The interface between emotion and motor control depends on interconnections among brain areas such as the amygdala, anterior cingulate cortex, and supplementary motor area (e.g., Devinsky et al., 1995; Oliveri et al., 2003). In the present study, state anxiety, HR, and GSR increased during the pressure test, suggesting that participants experienced increases in state anxiety and physiological arousal under pressure that contributed to the increased excitatory level of the CST.

The monetary reward and punishment for task performance (i.e., psychological pressure used in this study) may have increased mental effort and motivation. Motivation was defined as implicit psychological energy that directs human behavior and maintains it for a particular event (Hayamizu, 2008), and therefore psychological changes, such as increased mental effort and motivation, may have increased the excitatory level of the CST. Previous studies have reported that elevated motivation increases cortical excitabilities, based upon recordings of stimulus-preceding negativity using EEG (e.g., Kotani et al., 2003; Ohgami et al., 2006). In addition, many studies have examined the effects of psychological factors on CST excitation during the performance of motor skills. For instance, it has been demonstrated that motor imagery can increase CST excitability (e.g., Yahagi and Kasai, 1998). Further- more, it has been reported that directing participants' attention to their own movements while executing motor tasks increases CST excitability (Stefan et al., 2004). Thus, in the present study, it is reasonable to assume that CST excitation increased under pressure due to psychological variables such as enhanced mental effort and motivation.

Among the various performance indices, $\mathrm{AE}$ decreased significantly during the pressure test. Previous studies examining movement variability have indicated that decreases in intra-trial variability during performance of a continuous motor skill (Court et al., 2005) can be observed under psychological pressure, as can decreases in inter-trial variability during performance of a discrete motor skill (Higuchi et al., 2002). Gribble et al. (2003) indicated that movement accuracy during multi-joint limb movement leads to an increased level of co-contraction between agonists and antagonists along with a higher level of joint stiffness. Since the voluntary hand movement used in this study was designed to minimize movement accuracy, it can be assumed that the increased negative emotion and physiological arousal under pressure led to joint stiffness during the voluntary hand movements, and that this led to the observed decrease in AE. Furthermore, facilitated performance during the movement task could be due to the relatively low levels of stress response obtained in this study. Because there is thought to be an inverted-U relationship between physiological arousal and motor performance under pressure (Martens and Landers, 1970), it will be necessary to further examine the relationship amongst CST excitability, muscular activity, and motor performance of fine motor skills under a higher level of stress, such as during actual sports and daily stressful situations.

Additionally, the decreased AE under psychological pressure found in this study might be due to increased mental effort and motivation. The function of mental effort and motivation under pressure has been explained in the processing efficiency theory proposed by Eysenck and Calvo (1992) and the attentional control theory proposed by Eysenck et al. (2007). According to these theories, motor performance can improve or be maintained under pressure by elevating mental effort and motivation in the range of attentional processing resources. This explanation corroborates previous studies that have demonstrated an increase in mental effort when in- 
dividuals perform motor skills under pressure (e.g., Cooke et al., 2010; Williams et al., 2002).

While mean values for MEP variables and $\mathrm{AE}$ showed significant changes from the non-pressure to the pressure test, a significant positive correlation between MEP amplitude/bEMG and AE was also shown. This result shows that unchanged CST excitation under pressure facilitated performance accuracy of the fine motor task used in this study. Conversely, it also showed that increased CST excitation under pressure led to performance decrements in this task. Previous studies have reported that performance decrement or improvement under pressure relates to attentional shift involved in the conscious control of movements (Tanaka and Sekiya, 2011), increased arousal level (Martens and Landers, 1970), and kinematic changes (Ehrlenspiel et al., 2010). In the present study, findings suggest that CST excitability under pressure was also associated with changes in performance outcomes of motor skills.

This study is the first to provide evidence that excitatory levels of CST increase homogeneously during a fine motor task performed under psychological pressure. The results of this study suggest the importance of controlling CST excitability under psychological stress situations in realistic sports. Some psychological skill trainings, such as the control of attention and emotion, adjustment of physiological arousal, and motor imagery may play a key role in the control of CST excitation under stress. Future studies need to clarify the relationship between CST excitability under psychological stress and the effects of certain types of psychological skills trainings. Finally, two points concerning the limitation of this study should be mentioned. First, there is a limit to the range of motion when administering TMS because participants need to fix their head during the experiment in order to obtain correct data. Thus, it is difficult to apply TMS administrations to dynamic motion in sports or other physical activity. Second, there was a small sample size in this study. However, because activated CST excitability under pressure and the relationship between this excitation and task performance were clearly demonstrated despite the small sample size, findings from this study are informative for fields such as sports science, cognitive and emotional psychology, and neurophysiology.

\section{References}

Baumeister, R. F. (1984). Choking under pressure: Self-con- sciousness and paradoxical effects of incentives on skillful performance. J. Pers. Soc. Psychol., 46: 610-620.

Baumgartner, T., Willi, M., and Jäncke, L. (2007). Modulation of corticospinal activity by strong emotions evoked by pictures and classical music: a transcranial magnetic stimulation study. NeuroReport, 18: 261-265.

Beilock, S. L., and Carr, T. H. (2001). On the fragility of skilled performance: What governs choking under pressure? J. Exp. Psychol. Gen., 130: 701-725.

Capaday, C. (1997). Neurophysiological methods for studies of the motor system in freely moving human subjects. J. Neurosci. Methods, 74: 201-218.

Cooke, A., Kavussanu, M., McIntyle, D., and Ring, C. (2010). Psychological, muscular and kinematic factors mediate performance under pressure. Psychophysiology, 47: 1109-1118.

Coombes, S. A., Tandonnet, C., Fujiyama, H., Janelle, C. M., Cauraugh, J. H., and Summers, J. J. (2009). Emotion and motor preparation: A transcranial magnetic stimulation study of corticospinal motor tract excitability. Cogn. Affect Behav. Neurosci., 9: 380-388.

Court, M. L. J., Bennett, S. J., Williams, A. M., and Davids, K. (2005). Effects of attentional strategies and anxiety constraints on perceptual-motor organization of rhythmical arm movements. Neurosci. Lett., 384: 17-22.

Devanne, H., Lavoie, B. A., and Capaday, C. (1997). Inputoutput properties and gain changes in the human corticospinal pathway. Exp. Brain Res., 114: 329-338.

Devinsky, O., Morrell, M. J., and Vogt, B. A. (1995). Contributions of anterior cingulate cortex to behaviour. Brain, 118: 279-306.

Ehrlenspiel, F., Wei, K., and Sternad, D. (2010). Open-loop, closed-loop and compensatory control: performance improvement under pressure in a rhythmic task. Exp. Brain Res., 201: 729-741.

Eysenck, M. W., and Calvo, M. G. (1992). Anxiety and performance: The processing efficiency theory. Cogn. Emot., 6: 409-434.

Eysenck, M. W., Derakshan, N., Santos, R., and Calvo, M. G. (2007). Anxiety and cognitive performance: Attentional control theory. Emotion, 7: 336-353.

Gribble, P. L., Mullin, L. I., Cothros, N., and Mattar, A. (2003). Role of cocontraction in arm movement accuracy. J. Neurophysiol., 89: 2396-2405.

Hajcak, G., Molnar, C., George, M. S., Bolger, K., Koola, J., and Nahas, Z. (2007). Emotion facilitates action: a transcranial magnetic stimulation study of motor cortex excitability during picture viewing. Psychophysiology, 44: 91-97.

Hasegawa, Y., Kasai, T., Tsuji, T., and Yahagi, S. (2001). Further insight into the task-dependent excitability of motor evoked potentials in first dorsal interosseous muscle in humans. Exp. Brain Res., 140: 387-396.

Hasegawa, Y., Yano, M., Koyama, S., and Inomata, K. (2011). Golf putting under pressure: The effects of anxiety level and putting distance. Jpn. J. Sport Psychol., 38: 85-98 (in Japanese).

Hatfield, B. D. (2007). Cognitive neuroscience aspects of sport psychology: Brain mechanism underlying performance. In T. Morris, P. Terry, \& S. Gordon (eds.), Sport and Exercise Psychology: International Perspectives (pp. 121-137). Morgantown: Fitness Information Technology.

Hayamizu, T. (2008). Concept of motivation. In Japanese Society of Sport Psychology (ed.), Encyclopedia of Sport Psychology (pp. 237-243). Tokyo: Taishukan Shoten (in 
Japanese).

Hayashi, S., Hasegawa, Y., Yahagi, S., and Kasai, T. (2003). Effects of repetitive muscle contraction on excitability changes of human primary motor cortex. Japanese Journal of Physical Education, 48: 617-632 (in Japanese).

Hidano, N., Fukuhara, M., Iwawaki, M., Soga, S., and Speilberger, C. D. (2000). State-trait anxiety inventory-form JYZ. Tokyo: Japan UNI Agency (in Japanese).

Higuchi, T., Imanaka, K., and Hatayama, T. (2002). Freezing degrees of freedom under stress: Kinematic evidence of constrained movement strategies. Hum. Mov. Sci., 21: 831-846.

Kasai, T. (1993). Transcranial electric and magnetic stimulation in intact human subject. Jpn. J. Sports. Sci., 12: 54-70 (in Japanese).

Kazennikov, O., Solopova, I., Talis, V., and Ioffe, M. (2008). Anticipatory postural adjustment: The role of motor cortex in the natural and learned bimanual unloading. Exp. Brain Res., 186: 215-223.

Kotani, Y., Kishida, S., Hiraku, S., Suda, K., Ishii, M., and Aihara, Y. (2003). Effects of information and reward on stimulus-preceding negativity prior to feedback stimuli. Psychophysiology, 40: 818-826.

Liao, C., and Masters, R. S. W. (2002). Self-focused attention and performance failure under psychological stress. J. Sport Exerc. Psychol., 24: 289-305.

Martens, R., and Landers, D. M. (1970). Motor performance under stress: A test of the inverted-U hypothesis. J. Pers. Soc. Psychol., 16: 29-37.

Mullen, R., Hardy, L., and Tattersall, A. (2005). The effects of anxiety on motor performance: A test of the conscious processing hypothesis. J. Sport Exerc. Psychol., 27: 212-225.

Murray, N. P., and Janelle, C. M. (2007). Event-related potential evidence for the processing efficiency theory. J. Sports Sci., 25: 161-171.

Oathes, D., Bruce, J. M., and Nitschke, J. B. (2008). Worry facilitates corticospinal motor response to transcranial magnetic stimulation. Depression and Anxiety, 25: 969-976.

Ohgami, Y., Kotani, Y., Tsukamoto, T., Omura, K., Inoue, Y., Aihara, Y., et al. (2006). Effects of monetary reward and punishment on stimulus-preceding negativity. Psychophysiology, 43: 227-236.

Oliveri, M., Babiloni, C., Filippi, M. M., Caltagirone, C., Babiloni, F., Cicinelli, P., et al. (2003). Influence of the supplementary motor area on primary motor cortex excitability during movements triggered by neutral or emotionally unpleasant visual cues. Exp. Brain Res., 149: 214-221.

Rollnik, J. D., Schubert, M., and Dengler, R. (2000). Effects of a competitive stressor on motor cortex excitability: a pilot study. Stress Med., 16: 49-54.

Salvador, A., Suay, F., González-Bono, E., and Serrano, M. A. (2003). Anticipatory cortisol, testosterone and psychological responses to judo competition in young men. Psychoneuroendocrinology, 28: 364-375.

Stefan, K., Wycislo, M., and Classen, J. (2004). Modulation of associative human motor cortical plasticity by attention. J. Neurophysiol., 92: 66-72.

Tanaka, Y., Funase, K., Sekiya, H., Sasaki, J., and Takemoto, T. (2011). Multiple EMG activity and intracortical inhibition and facilitation during a fine finger movement under pressure. J. Mot. Behav., 43: 73-81.

Tanaka, Y., and Sekiya, H. (2010). The influence of audience and monetary reward on the putting kinematics of expert and novice golfers. Res. Q. Excerc. Sport, 81: 416-424.
Tanaka, Y., and Sekiya, H. (2011). The influence of monetary reward and punishment on psychological, physiological, behavioral, and performance aspects of a golf putting task. Hum. Mov. Sci, 30: 1115-1128.

van Galen, G. P., Muller, M., Meulenbroek, R. G. J., and van Gemmert, A. W. A. (2002). Forearm EMG response activity during motor performance in individuals prone to increased stress reactivity. Am. J. Ind. Med., 41: 406-419.

van Loon, A. M., van den Wildenberg, W. P. M., van Stegeren, A. H., Hajcak, G., and Ridderinkhof, K. R. (2010). Emotional stimuli modulate readiness for action: A transcranial magnetic stimulation study. Cogn. Affect Behav. Neurosci., 10: $174-181$.

van Loon, E. M., Masters, R. S. W., Ring, C., and McIntyre, D. B. (2001). Changes in limb stiffness under conditions of mental stress. J. Mot. Behav., 33: 153-164.

Vickers, J. N., and Williams, A. M. (2007). Performing under pressure: The effects of physiological arousal, cognitive anxiety, and gaze in biathlon. J. Mot. Behav., 39, 381-394.

Williams, A. M., Vickers, J., and Rodrigues, S. (2002). The effects of anxiety on visual search, movement, and performance in table tennis: A test of Eysenck and Calvo's processing efficiency theory. J. Sport Exerc. Psychol., 24: 438-455.

Yahagi, S., and Kasai, T. (1998). Facilitation of motor evoked potentials (MEPs) in first dorsal interosseous (FDI) muscle is dependent on different motor images. Electroencephalogr. Clin. Neurophysiol., 109: 409-417.

Yahagi, S., Takeda, Y., Ni, Z., Takahashi, M., Tsuji, T., Komiyama, T., et al. (2005). Modulations of input-output properties of corticospinal tract neurons by repetitive dynamic index finger abductions. Exp. Brain Res., 161: 255-264.

Yoshie, M., Kudo, K., Murakoshi, T., and Ohtsuki, T. (2009). Music performance anxiety in skilled pianists: effects of social-evaluative performance situation on subjective, autonomic, and electromyographic reactions. Exp. Brain Res., 199: 117-126.

Yoshie, M., Kudo, K., and Ohtsuki, T. (2008). Effects of psychological stress on state anxiety, electromyographic activity, and arpeggio performance in pianists. Med. Probl. Perform. Artists, 23: 120-132. 
Name:

Yoshifumi Tanaka

\section{Affiliation:}

Faculty of Education and Regional Studies, University of Fukui

\section{Address:}

3-9-1 Bunkyo, Fukui, 910-8507 JAPAN

\section{Brief Biographical History:}

1996-2000 Undergraduate Program, Faculty of Integrated Arts and Sciences, Hiroshima University

2000-2003 Teacher, Nagoya Resort and Sport College 2003-2005 Master's Program, Graduate School of Biosphere Science, Hiroshima University

2005-2008 Doctoral Program, Graduate School of Biosphere Science, Hiroshima University

2008 Received Ph.D. at Hiroshima University

2009-2012 Lecturer, Faculty of Economics, Tezukayama University

2012- Lecturer, Faculty of Education and Regional Studies, University of Fukui

Main Works:

- Tanaka, Y., and Sekiya, H. (2011). The influence of monetary reward and punishment on psychological, physiolosical, behavioral, and performance aspects of a golf putting task. $\mathrm{Hu}$ man Movement Sceince, 30, 1115-1128.

-Tanaka, Y., Funase, K., Sekiya, H., Sasaki, J., and Takemoto, T. (2011). Multiple EMG activity and intracortical inhibition and facilitation during a fine finger movement under pressure. Journal of Motor Behavior, 43, 73-81.

-Tanaka, Y., and Sekiya, H. (2010). The influence of audience and monetary reward on the putting kinematics of expert and novice golfers. Research Quarterly for Exercise and Sport, 81, 416-424.

-Tanaka, Y., and Sekiya, H. (2010). The relationships between psychological/physiological changes and behavioral/performance changes in a golf putting task under pressure. International Journal of Sport and Health Science, 8, 83-94.

\section{Membership in Learned Societies:}

-International Society of Sport Psychology

- North American Society for Psychology of Sport and Physical Activity

- Japan Society of Physical Education, and Health and Sport Science

- Japanese Society of Sport Psychology

- The Japan Neuroscience Society 